\title{
Distinct Roles for Alpha- and Beta-Band Oscillations during Mental Simulation of Goal-Directed Actions
}

\author{
Loek Brinkman, ${ }^{1}$ Arjen Stolk, ${ }^{1}$ H. Chris Dijkerman, ${ }^{2}$ Floris P. de Lange, ${ }^{1}$ and $\oplus^{-I v a n ~ T o n i ~}{ }^{1}$ \\ ${ }^{1}$ Radboud University Nijmegen, Donders Institute for Brain, Cognition and Behaviour, 6500 HB Nijmegen, The Netherlands and ${ }^{2}$ Utrecht University, \\ Helmholz Institute, Experimental Psychology, 3584 CS Utrecht, The Netherlands
}

\begin{abstract}
Rhythmic neural activity within the alpha $(8-12 \mathrm{~Hz})$ and beta $(15-25 \mathrm{~Hz})$ frequency bands is modulated during actual and imagined movements. Changes in these rhythms provide a mechanism to select relevant neuronal populations, although the relative contributions of these rhythms remain unclear. Here we use MEG to investigate changes in oscillatory power while healthy human participants imagined grasping a cylinder oriented at different angles. This paradigm allowed us to study the neural signals involved in the simulation of a movement in the absence of signals related to motor execution and sensory reafference. Movement selection demands were manipulated by exploiting the fact that some object orientations evoke consistent grasping movements, whereas others are compatible with both overhand and underhand grasping. By modulating task demands, we show a functional dissociation of the alpha- and beta-band rhythms. As movement selection demands increased, alpha-band oscillatory power increased in the sensorimotor cortex ipsilateral to the arm used for imagery, whereas beta-band power concurrently decreased in the contralateral sensorimotor cortex. The same pattern emerged when motor imagery trials were compared with a control condition, providing converging evidence for the functional dissociation of the two rhythms. These observations call for a re-evaluation of the role of sensorimotor rhythms. We propose that neural oscillations in the alpha-band mediate the allocation of computational resources by disengaging task-irrelevant cortical regions. In contrast, the reduction of neural oscillations in the beta-band is directly related to the disinhibition of neuronal populations involved in the computations of movement parameters.
\end{abstract}

Key words: efference copy; magnetoencephalography; motor imagery; motor plan; movement preparation; sensorimotor cortex

\section{Introduction}

To interact with our environment, we construct action plans to issue appropriate motor commands to our body. It has been suggested that action plans are specified and evaluated by mentally simulating the action to predict the sensory consequences of that particular movement (Wolpert and Ghahramani, 2000; Davidson and Wolpert, 2005; Shadmehr and Krakauer, 2008). During mental simulation of an action, specific neuronal populations controlling particular features of that movement need to be selected, while other neuronal populations need to be suppressed. Here we assess a mechanism for implementing these processes of activation and suppression in the sensorimotor system, based on neuronal oscillations in the alpha $(8-12 \mathrm{~Hz})$ and beta $(15-25 \mathrm{~Hz})$ band.

Processes that involve the mental simulation of actions have consistently been associated with a reduction of oscillatory power

\footnotetext{
Received May 19, 2014; revised Aug. 21, 2014; accepted Sept. 25, 2014.

Author contributions: L.B., A.S., C.D., F.P.d.L., and I.T. designed research; L.B. performed research; A.S. contributed unpublished reagents/analytic tools; L.B. analyzed data; L.B. and I.T. wrote the paper.

This work was supported by the Netherlands Organisation for Scientific Research (NWO Brain and Cognition 433-09-248).

The authors declare no competing financial interests.

This article is freely available online through the J Neurosci Author Open Choice option.

Correspondence should be addressed to Loek Brinkman, Radboud University Nijmegen, Donders Institute

for Brain, Cognition and Behaviour, P.O. Box 9101, 6500 HB Nijmegen, The Netherlands. E-mail: L.Brinkman@donders.ru.nl.

DOI:10.1523/JNEUROSCI.2039-14.2014

Copyright $\odot 2014$ the authors $\quad 0270-6474 / 14 / 3414783-10 \$ 15.00 / 0$
}

in both the alpha and beta frequency bands over sensorimotor regions in both humans and nonhuman primates (Sanes and Donoghue, 1993; Mackay, 1997; Pfurtscheller and Lopes da Silva, 1999; McFarland et al., 2000; Jurkiewicz et al., 2006; de Lange et al., 2008). A reduction in oscillatory power in either frequency band corresponds to the disinhibition of a cortical region (Mackay, 1997; van Wijk et al., 2012; Cheyne, 2013). Although oscillatory power in the alpha- and beta-bands is highly correlated (Carlqvist et al., 2005; de Lange et al., 2008), there is also evidence that these rhythms serve distinct functions. For instance, the cortical beta rhythm is coherent with the EMG of the muscles, whereas corticomuscular coherence in the alpha-band is largely absent (Mima and Hallett, 1999; Brown, 2000; Kilner et al., 2000; van Ede and Maris, 2013). Furthermore, beta-band power changes are somatotopically organized, whereas alpha-band power changes are not effector specific (Salmelin et al., 1995; Crone et al., 1998; Nierula et al., 2013). These findings suggest that neural oscillations in the alpha- and beta-bands support mental simulation of actions with different functional mechanisms. Here we test this hypothesis by recording oscillatory neural activity in humans with MEG during performance of a motor imagery task. Motor imagery allows one to measure neural signals related to the simulation of an action in the absence of movement-related signals and somatosensory reafference (Jeannerod, 1994; Jeannerod and Decety, 1995). Participants were asked to imagine grasping a cylinder oriented at different angles. 
Movement selection demands were manipulated by exploiting the fact that some object orientations evoke consistent grasping movements, whereas other orientations are compatible with both overhand and underhand grasping (Rosenbaum and Jorgensen, 1992; Cisek and Kalaska, 2010; Wood and Goodale, 2011). Neural oscillatory effects associated with motor imagery were isolated using a two-tier approach. First, we tested how changes in oscillatory power evoked during imagery where modulated by task demand. Second, we used a subtractive logic between different tasks. Changes in oscillatory power observed during the motor imagery task were compared with the changes observed during a control task that used the same visual input and response contingencies, but where no imagery was required. The two approaches show converging evidence that while beta-band activity is involved in the disinhibition of neuronal population in the contralateral sensorimotor cortex, alpha-band activity is involved in the inhibition of cortical regions irrelevant for the task at hand.

\section{Materials and Methods}

Participants. In the first experiment (behavior only), 12 healthy, righthanded human participants (age: $23 \pm 1.5,1$ male) performed a reaction time version of a motor imagery task.

In the second and main experiment (MEG and behavior), 27 healthy, right-handed human participants (age: $23 \pm 0.5$ years, mean \pm SE, 10 males) performed a motor imagery and a control task, using a delayed response. Three participants who did not comply with the task were excluded from further analyses (see Results), resulting in a total number of 24 datasets. The study was approved by the local ethics committee and a written informed consent was obtained from the subjects according to the Declaration of Helsinki.

Experimental design and procedure. This study comprised two experiments. In the first experiment we assessed the duration of goal-directed motor imagery of grasping movements. In the second and main experiment, we measured changes in neural activity associated with the imagery of those movements using MEG. To keep the imagery process in both experiments as similar as possible, both experiments were performed while participants sat upright in an illuminated, magnetically shielded room facing a projection screen.

In the first experiment, participants performed a reaction time version of the motor imagery task. In this task, a black-white cylinder was presented at the center of the screen and participants were asked to imagine grasping the middle-third of the cylinder using whole-hand prehension (Fig. 1C). Each trial started with the presentation of a fixation cross on the center of a gray screen (range: 3000-4000 ms, randomly sampled from a uniform distribution) followed by the image of the cylinder (covering $13 \times 2.3^{\circ}$ of visual angle, projected on the center of the screen, Fig. $1 A)$. The cylinder consisted of a black part and a white part, with slightly different surface areas (e.g., 54\% black and $46 \%$ white, or vice versa, counterbalanced across trials). The difference in surface area was irrelevant for the imagery task, but was used in the control task of the second experiment (see below). Across trials, the cylinder's orientation on the transverse plane varied across 30 different possibilities, between 0 and $348^{\circ}$, in steps of $12^{\circ}$, with $0^{\circ}$ being the vertical position, and $36^{\circ}$ as the minimum orientation difference between two subsequent trials. As soon as participants finished imagining the grasping movement, they reported whether their thumb was on the black or the white part of the cylinder by saying out loud either "black" or "white." The interval between the presentation of the stimulus and the onset of the verbal response provided a measure of the duration of the imagery process. We used a verbal response to avoid the confound of spatial congruency effects between the location of the thumb relative to the cylinder and the location of a button press relative to the body. The presentation of the stimuli and the detection of the onset of the verbal responses were controlled by Presentation 14.9 (Neurobehavioral Systems). Participants completed 480 trials of this task. Trials in which the automatic voice-onset detection was incorrect (e.g., premature triggering by noise or failure of triggering if verbal re- sponse was not loud enough) were excluded from the analysis ( $1 \pm 1 \%$, mean percentage of rejected trials $\pm \mathrm{SE}$ ).

In the second and main experiment of this study, we measured changes in neural activity associated with the imagined grasping movements. Participants performed the same motor imagery task, but now used a delayed response to exclude the possibility that preparing to report the decision could influence cerebral activity evoked during performance of the imagery task. On each trial, the cylinder was replaced after $1500 \mathrm{~ms}$ by a response screen consisting of two squares (one black and one white, $2 \times$ $2^{\circ}$ of visual angle) located at the left and right side of the horizontal center plane (11.6 $6^{\circ}$ of visual angle apart), and were presented until a response was made. Participants reported black or white by pressing one of two buttons with the index finger of their left or right hand, respectively, according to the relative location of corresponding white/black squares on the response screen (Fig. 1E). Because the relative location of the black and white squares on the response screen was pseudorandomized, participants could only decide which finger to use for reporting their decision after presentation of the response screen, excluding the possibility that preparing to report the decision could influence cerebral activity during performance of the imagery task. During the motor imagery task, participants were instructed beforehand on which hand they would imagine moving. This instruction changed every 10 trials over 48 blocks, for a total of 480 trials ( 240 trials per hand).

In addition, in the main experiment participants also performed a control task (order counterbalanced across participants). In the control task, which used the same visual input and response contingencies, participants reported which side of the stimulus was larger (black or white). The control task allowed the correction of neural changes unrelated to the imagery process, such as those evoked by the visual input. Participants performed 240 trials of the control task ( 480 for four participants). In both experiments, tasks included short breaks every 60 trials, where participants indicated themselves when they were ready to continue (typically after 1-2 $\mathrm{min})$.

Data acquisition. Ongoing brain activity was recorded continuously using a whole-head MEG system with 275 axial gradiometers (VSM/CTF system, $300 \mathrm{~Hz}$ low-pass filter, $1200 \mathrm{~Hz}$ sampling rate). The participant's head position with respect to the MEG sensor array was monitored during the course of the experiment using localization coils attached to the anatomical landmarks, i.e., the nasion and left and right ear canals (Stolk et al., 2013). To correct for eye and actual arm movements in the off-line analysis, we also recorded EOG and EMG using a bipolar montage of 10 $\mathrm{mm} \mathrm{Ag-AgCl}$ surface electrodes placed on the supraorbital and infraorbital ridge of the left eye (vertical EOG) and on the outer canthi (horizontal EOG), and on the left and right forearms approximately above the musculus brachioradialis and the musculus flexor digitorum superficialis. EMG signals were high-pass filtered $(20 \mathrm{~Hz})$, rectified, and integrated (root mean square) over the task interval $(0-1500 \mathrm{~ms}$ after stimulus presentation). Structural T1 MRI scans for forward modeling were obtained for all subjects on either a Siemens 1.5T Avanto (TR/TE 2250 ms/2950 ms) or a Siemens 3 T Trio MRI scanner (TR/TE 2300 ms/3030 $\mathrm{ms}$ ). During MR acquisition, identical earplugs (now with a vitamin $\mathrm{E}$ capsules in place of the MEG localization coils) were used for coregistration of the MRI and MEG data.

Data analysis. In the motor imagery task, we tested whether the reports of the participants were sensitive to the biomechanical constraints imposed by the anatomy of the left and right arms. These constraints predict that the preferred manner in which the cylinder would be grasped with either hand would be modulated by the orientation of the stimulus, and would give rise to two orientations where the preferred grasping switches from the white to the black side of the cylinder (switch points). For imagery with the left hand, these switch-point orientations are expected to be in the bottom-right and top-left quadrant $\left(90-180^{\circ}\right.$ and $270-360^{\circ}$, respectively). Imagined grasping with the right hand should give rise to similar switch points at orientations that are mirror images of those found for left-hand imagery, in the top-right and bottom-left quadrants $\left(0-90^{\circ}\right.$ and $180-270^{\circ}$, respectively). Stimuli with orientations around the switch points could potentially be grasped using two different hand configurations (thumb either on the black or the white part of the cylinder). We reason that these stimulus orientations would place a higher 
demand on the selection process of the action, as a decision has to be made between two possible actions (Fig. 1B). In contrast, stimulus orientations orthogonal to the switch points show a clear preferred hand configuration in which they are grasped and hence place a lower demand on the process of action selection (Fig. 1B). The relative task demand of each orientation can therefore be expressed as the trial-by-trial variability in which the stimulus is grasped. The orientations of the switch points were defined for each participant separately by fitting a sine wave to the behavioral data, where the zero-crossing of the sine wave determined the switch points. To obtain robust estimates of the effect of task demand on MEG data, we define "high-demand" stimuli as those stimuli oriented around the switch points (range: four orientation bins, i.e., -24 to $+24^{\circ}$ ) and compared those stimuli orientations to "low-demand" orientations, orthogonal to the switch points and covering an equivalent range.

MEG data analysis was performed using the Fieldtrip toolbox (Oostenveld et al., 2011) and custom MATLAB code (Matlab 7.9; The MathWorks). The preprocessing of the data consisted of three steps. First, trials containing large transients (e.g., due to sensor noise or SQUID jumps) or muscle artifacts were removed using a semi-automatic procedure with an adaptive threshold based on the $z$-scores of the data [large transients: cutoff $z$-value $50 \pm 5$, mean \pm SE; muscle artifacts: bandpass filtered data $(110-140 \mathrm{~Hz}$, i.e., a frequency band containing most muscle-related effects), cutoff $z$-value $16 \pm 1$, mean \pm SE].

Next, all trials were visually inspected and trials that contained residual artifacts were removed from the data. Finally, trials that showed EMG activity during the imagery interval (defined as EMG signals larger than two SDs from the root mean square of EMG signals for each subject and condition) were removed, resulting in $82 \pm 4 \%$ (mean \pm SE) of the original trials included for further analysis.

MEG source reconstruction and spectral analysis. The neural sources generating the sensor-level MEG data were reconstructed by adopting two beamforming techniques to either extract the topographical distribution of a time- and frequency-range of interest (dynamic imaging of coherent sources, DICS; Gross et al., 2001; Liljeström et al., 2005) or to estimate the MEG time series at a topographical region of interest for subsequent spectral analysis (linearly constraint minimum variance, LCMV; van Veen et al., 1997). Participant-specific anatomical MRIs were used to linearly transform a 3D template grid (1 cm spacing) in MNI coordinates to the coordinates system specific to the participant's head. We subsequently applied the inverse of this transformation to obtain grid points at matched brain locations across participants. Spatial filters were constructed for each of the grid positions, passing the activity from the location of interest with unit gain, while maximally suppressing activity from all other possible sources of neural and non-neural activity. The DICS spatial filter is constructed from the lead field and the crossspectral density matrix of the data. The lead field is the physical forward model of the field distribution calculated from an assumed source at a given location and the participant-specific volume conduction model of the head. Here, we used a single-shell volume-conduction model of the brain, based on the brain boundaries determined by the segmented anatomical MRI to compute the lead field (Nolte, 2003). Source localization using the DICS beamforming approach was performed separately for the different frequency bands (alpha: $7.5-12.5 \mathrm{~Hz}$; beta: $15-25 \mathrm{~Hz}$; and a frequency range encompassing both the alpha- and beta-bands: 7.5-25 $\mathrm{Hz}$ ) for a window of interest during stimulus presentation (600-1000 $\mathrm{ms}$ ) and for a prestimulus baseline interval ( -600 to $-200 \mathrm{~ms})$, using a common spatial filter based on the pooled data from both time intervals. The interval of 600-1000 ms was selected for showing the largest power difference between left- and right-hand imagery at the sensor level (orthogonal to all subsequent analyses). We estimated the spatial topographies of source power separately for all conditions (left-hand imagery, right-hand imagery, and control condition) and subsequently contrasted the power estimates between all points in our grid (i.e., all voxels). Two regions of interest were selected by contrasting the spatial topographies of left- and right-hand imagery over a frequency range encompassing both alpha- and beta-bands. We selected the voxels that showed the largest difference between the two conditions as regions of interest. The location of these voxels corresponded to the left and right sensorimotor cortices (MNI coordinates: [42 -30 60] and [ $-50-3848]$ ). To obtain the time courses at these spatial locations, the sensor-level time courses were projected through two spatial filters, corresponding to the two regions of interest. These spatial filters were obtained using an LCMV beamforming approach, which is identical to the DICS beamforming approach, but uses the covariance matrix of the sensor level MEG data instead of the cross-spectral density matrix. After reconstructing the sensor-level time series at the location of the sensorimotor cortices, timefrequency representations (TFRs) were calculated over a frequency range of 5-40 Hz using a Fourier transform approach. Each data segment was multiplied with a Hanning taper and spectral power was estimated every $50 \mathrm{~ms}$ using a $400 \mathrm{~ms}$ sliding window with a step size of $1.25 \mathrm{~Hz}$ (resulting in $5 \mathrm{~Hz}$ frequency smoothing). All power spectra were baseline corrected and expressed as relative changes compared with a prestimulus baseline interval (baseline period: -600 to $-200 \mathrm{~ms}$ before stimulus onset). The relevant trials for each analysis were averaged by taking the median of the power spectra (within subjects). For visualization purposes, line plots have been smoothed using a weighted sliding window, averaging over a range of \pm 2 data points (smoothing kernel: $\left[1 / 32 / 31^{2 / 3} 1 / 3\right]$ ) and TFRs have been interpolated and smoothed using Fieldtrip.

Statistical inferences of MEG data. We considered two differential effects during corresponding events in the motor imagery and control conditions: (1) modulation of imagery-related signals by selection demand, and (2) imagery versus the control condition. The reliability of these differential effects was tested using nonparametric, cluster-based permutation statistics as a means to control for multiple comparisons, while retaining optimal sensitivity (Maris and Oostenveld, 2007; Maris, 2012). This procedure entails three steps. First, separate paired-sample $t$ tests are performed for all data points between the two conditions of interest. Second, clusters of adjacent data points (adjacent in time, space, and/or frequency) are defined by means of a clustering algorithm using a threshold of $p<0.05$. Finally, these clusters are evaluated against a permutation distribution, obtained by 10,000 permutations of randomly shuffling the conditions within all participants, resulting in a Monte Carlo $p$ value. Because the power changes for left- and right-hand imagery were symmetrical, left- and right-hand imagery trials were combined by considering the sensorimotor cortices as ipsilateral and contralateral to the effector used for imagery to gain maximal sensitivity. In the control condition, the power over the sensorimotor cortices was averaged over the left and right hemispheres. For statistical inference, clusters were defined using time $(0-1500 \mathrm{~ms})$ and frequency (alpha $8-12 \mathrm{~Hz}$, beta $15-25 \mathrm{~Hz}$ ) as clustering dimension. All statistical tests were performed separately for the alpha and beta frequency ranges, allowing for datadriven clusters within the frequency bands.

The modulatory effect of task demand was also assessed by evaluating whether the spectral power during imagery (averaged over 0-1500 ms after stimulus presentation) was correlated with task demands. We computed vectors for each subject describing the spectral power and relative task demand as a function of stimulus orientation. After applying smoothing to both the behavioral and the spectral data to reveal the underlying patterns (weighted moving sliding window average over a

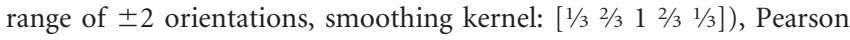
correlation coefficients were calculated between task demand and spectral power for both hands. This procedure resulted in four correlation coefficients per subject: 1 ("task demand") $\times 2$ ("hemisphere": ipsilateral, contralateral) $\times 2$ ("frequency": alpha, beta). The correlation coefficients were tested to deviate from zero by a two-sided $t$ test and the functional dissociation of alpha- and beta-band rhythms was tested by a two-way repeatedmeasure ANOVA on the absolute values of the correlation coefficients (2 (frequency: alpha, beta) $\times 2$ (hemisphere: ipsilateral, contralateral)).

\section{Results}

\section{Task performance}

In the first experiment we assessed the duration of the imagery process using a reaction time version of the imagery task. Participants imagined grasping the cylinder with either their left or right hand and, as soon as the movement was completed, indicated with a verbal response which part of the cylinder was held by their thumb (black or white). Imagining those movements 
A

A First experiment behaviour only

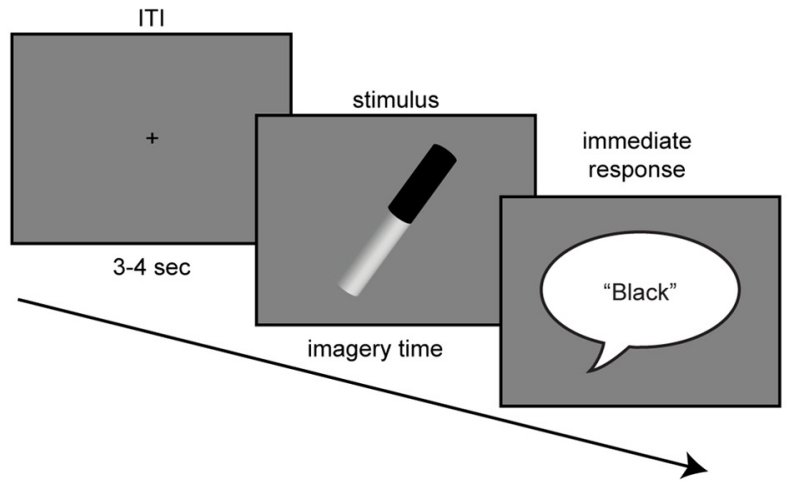

C Low demand trials
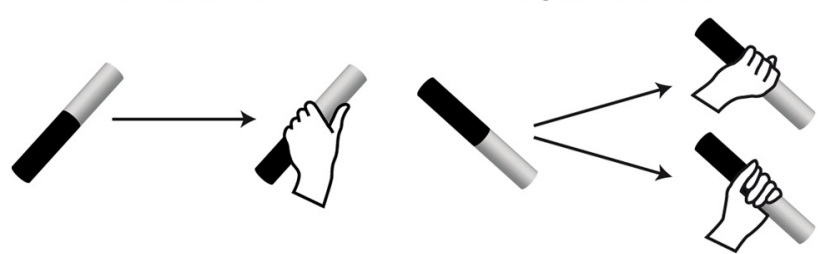

E

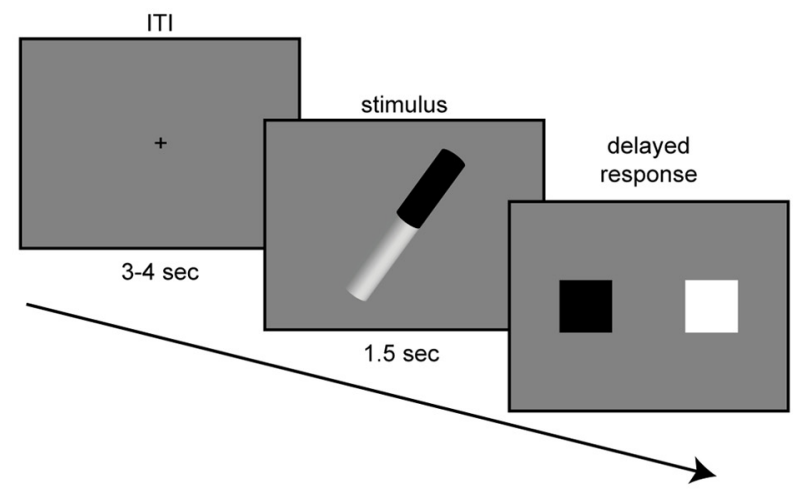

B

First experiment

behaviour only

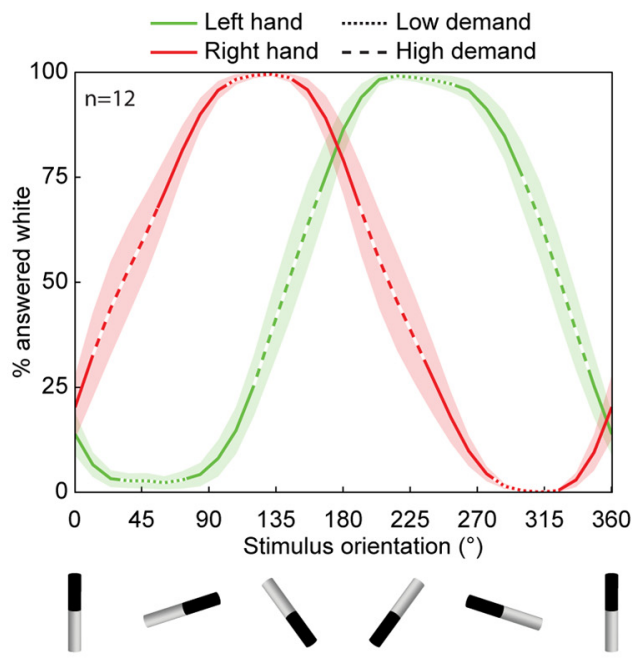

D

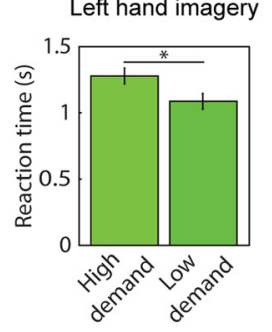

F

Second experiment

MEG \& behaviour

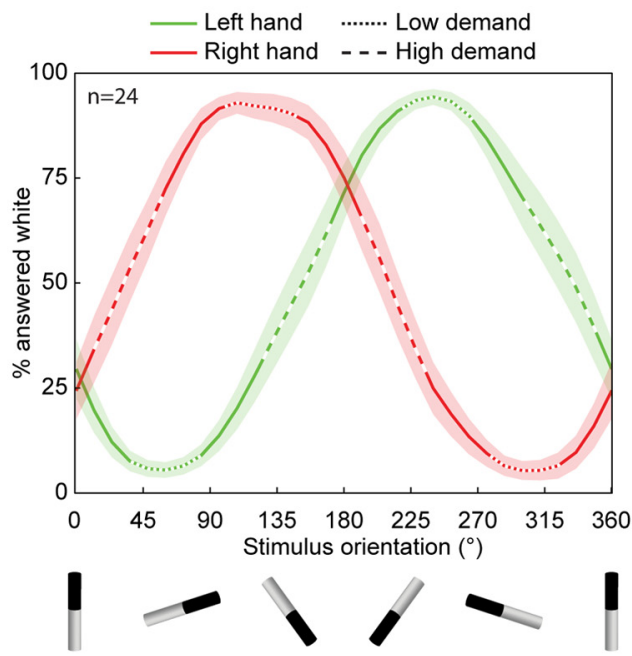

Figure 1. Task design and behavioral performance. $A$, In the first experiment, participants performed a reaction time version of the motor imagery task. Each trial started with a fixation cross (ITI, intertrial interval), followed by an image of a tilted cylinder. Participants imagined whole-hand prehension of the cylinder with either the left or right hand (blocked). As soon as participants finished imagining the grasping movement, they reported whether their thumb was on the black or the white part of the cylinder by saying out loud either black or white. Some stimulus orientations afforded two ways in which the cylinder could be grasped, which were more demanding in terms of selecting an action plan, compared with orientations that afforded only one grip ( $)$. B, Percentage of trials in which participants reported to have imagined grasping thecylinder with theirthumb on the white part, as afunction of cylinder orientations, during trials involving theleftand the right hand (green and red curves, respectively). The dashed segments of the linesindicatethe regimes of stimulus orientations of high-and low-demand trials. Shaded areas indicate \pm 1 SE. High-demand trials were consistently associated with longer reaction times ( $\boldsymbol{D}$; mean reaction time \pm 0.5 SE of the difference between conditions; ${ }^{*} p<0.0005$, paired-samplettest).E, In thesecond experiment, participants performed adelayed-responseversion of themotorimagerytask. After 1.5 sthecylinder was replaced with aresponsescreen containing a black and a white square in pseudorandomized order. The overall pattern of the responses in the delayed-response version of the task was very similar to that of the reaction time version of the task $(\boldsymbol{B}, \boldsymbol{F})$.

took $1165 \pm 80 \mathrm{~ms}$ and $1110 \pm 80 \mathrm{~ms}$, for left and right hand, respectively (mean $\pm \mathrm{SE}$ ). For each hand, there were stimulus orientations that participants reported grasping with underhand and overhand postures equally frequently (switch points). We label these and their neighboring orientations ( \pm 24 degrees around the switch points) as high-demand orientations, given that in these trials participants needed to select from two possible actions (Fig. $1 B, C$ ). Similarly, low-demand orientations, which 
showed a clear preferred manner in which they were grasped, were selected orthogonal to the high-demand orientations (Fig. $1 B, C)$. Task performance indicated that high-demand trials took longer to solve than low-demand trials (Fig. 1D). The difference in reaction time between high- and low-demand trials for the left hand was $190 \pm 60 \mathrm{~ms}\left(t_{(11)}=5.5, p<0.0005\right)$ and for the right hand was $155 \pm 95 \mathrm{~ms}\left(t_{(11)}=5.8, p<0.0005\right.$; mean $\pm \mathrm{SE}, p$ values of paired-sample $t$ tests). Furthermore, the switch points were shifted by $180^{\circ}$ depending on the hand the participants were asked to imagine moving (left hand: $143 \pm 6$ and $323 \pm 6^{\circ}$; right hand: $34 \pm 8$ and $213 \pm 8^{\circ}$, mean switch point \pm SE; Fig. $1 B$ ), in line with the biomechanical constraints of the left and right arms (Fig. 1C). To ascertain that participants were engaged in motor imagery for the entire course of the experiment, we split the behavioral data in four temporal windows (epochs). Both the differences between low- and high-demand trials as well as the switch point were present across all epochs.

In the second and main experiment of this study, we recorded neural changes associated with the imagery process using MEG. A delayed response was introduced to exclude the possibility that preparing to report the decision could influence cerebral activity evoked during performance of the imagery task. During the delayed-response version of the imagery task, participants showed a pattern of switch points similar to that observed in the first experiment (left hand: $150 \pm 8$ and $326 \pm 7^{\circ}$; right hand: $36 \pm 6$ and $214 \pm 6^{\circ}$, mean switch point \pm SE) present across the whole experiment. This behavioral pattern was consistently found in all but three participants. As these three participants did not consider biomechanical constraints during task performance, their data were excluded from further analyses. Additionally, participants performed a control task using the same visual input and response contingencies, where participants reported which side of the stimulus was larger (black or white). The control task allowed correction for neural changes unrelated to the imagery process, such as those evoked by the visual input. In the control task participants committed very few errors ( $98 \pm 4 \%$ correct, mean \pm SE).

\section{Dissociation of alpha and beta: modulation by task demand}

While participants were engaged in motor imagery, power in the alpha- and beta-bands decreased over occipital, parietal, and sensorimotor regions. In both frequency ranges, the power decrease over the sensorimotor cortex was strongest for the hemisphere contralateral to the hand used for the imagined movement. Therefore, contrasting left- and right-hand imagery trials gave rise to similar lateralized power distributions over the sensorimotor cortices for both rhythms (Fig. 2A,B). To investigate whether the alpha- and beta-band rhythms could be functionally dissociated, we assessed how task demand modulated the evoked power changes in both frequency ranges. When subjects imagined grasping objects that afforded two grip types (high-demand trials) the alpha-band power in the ipsilateral sensorimotor sensors was significantly higher compared with the low-demand trials that afforded only one grip type (Fig. 2C,E; $104 \pm 2$ trials per subject per condition, mean $\pm \mathrm{SE}$ ). Cluster-based permutation statistics (using time and frequency as clustering dimensions) showed this effect to be present in the alpha-band $(10-12.5 \mathrm{~Hz})$ between 700 and $1250 \mathrm{~ms}$ after stimulus presentation $(p<0.01)$, within the time frame of the imagined movement (Fig. 1D). Concurrently, beta-band activity showed a stronger power decrease for high-demand trials over the contralateral sensorimotor sensors (Fig. 2D,F). This modulation was present in the beta-band $(16-20 \mathrm{~Hz})$ between 1100 and $1300 \mathrm{~ms}$ after stimulus presentation $(p<0.05)$, within the time frame of the imagined movement
(Fig. 1D). The dissociation of the alpha- and beta-band rhythms was further qualified by correlating the relative task demand as a function of stimulus orientation to the alpha- and beta-band power in the ipsilateral and contralateral sensorimotor cortices in those trials (averaged over $0-1500 \mathrm{~ms}$ after stimulus presentation). Alpha-band power in the ipsilateral sensorimotor cortex showed a positive correlation with task demands, whereas betaband power in the contralateral sensorimotor cortex showed a negative correlation with task demands (Fig. $2 E, F$, insets; ipsilateral alpha: $-0.12 \pm 0.06, t_{(23)}=2.3, p<0.5$; contralateral beta: $0.14 \pm 0.06, t_{(23)}=-2.1, p<0.5$; average Pearson correlation coefficients $\pm \mathrm{SE}, p$ values of two-sided $t$ tests). The dissociation of the alpha- and beta-band rhythms was apparent from the significant interaction of frequency and hemisphere (2 (frequency: alpha, beta) $\times 2$ (hemisphere: ipsilateral, contralateral) two-way repeated-measure ANOVA on the absolute values of the correlation coefficients, $\left.F_{(1,23)}=4.4, p<0.05\right)$.

\section{Dissociation of alpha and beta: neural characteristics of imagery and control}

The second approach to investigate the functional dissociation of alpha- and beta-band rhythms was to compare the evoked power changes during motor imagery to a control condition. Using this approach, we correct for power changes unrelated to the imagery process, such as those evoked by the presentation of the stimuli or preparing a response. In the control condition, participants were presented with the same visual input, but instead of imagining grasping the cylinder, participants judged whether the black or the white part of the stimulus was largest. While participants were engaged in the control task, power in the alpha- and betabands decreased over occipital, parietal, and sensorimotor regions. Relative to the control task, the dynamics of the sensorimotor power changes evoked during motor imagery were qualitatively different across the alpha- and beta-bands. In the alpha-band, the dynamics of the control condition closely matched the dynamics observed in the sensorimotor cortex contralateral to the imagined hand movements (Fig. 3C). Put differently, motor imagery increased alpha-band power in the sensorimotor cortex ipsilateral to the imagined hand, as compared with control. Cluster-based permutation statistics (using time and frequency as clustering dimensions) showed that this increase in oscillatory power occurred in the alpha-band (9-12.5 $\mathrm{Hz}$ ) between 350 and $1150 \mathrm{~ms}$ after stimulus presentation (Fig. $3 A ; p<0.01$ ), within the time frame of the imagined movement (Fig. 1D). In the beta-band, the dynamics of the control condition initially matched the sharp power decrease observed also during motor imagery of the contralateral hand, but later showed an equally sharp rebound to baseline values, matching more closely the power values observed during motor imagery of the ipsilateral hand (Fig. 3D). Put differently, motor imagery decreased beta power in the sensorimotor cortex contralateral to the imagined hand, as compared with control. Cluster-based permutation statistics (using time and frequency as clustering dimensions) showed that this decrease in oscillatory power occurred in the beta-band $(15-20 \mathrm{~Hz})$ between 600 and $1050 \mathrm{~ms}$ after stimulus presentation (Fig. $3 B ; p<0.025$ ), within the time frame of the imagined movement (Fig. 1D).

\section{Discussion}

This study explored the relative contribution of alpha- and betaband oscillations during the mental simulation of goal-directed actions. There is one main finding, confirmed in two different experimental settings: during the mental simulation of an action, 
A

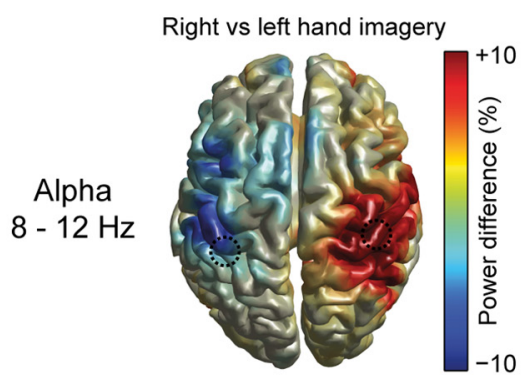

C

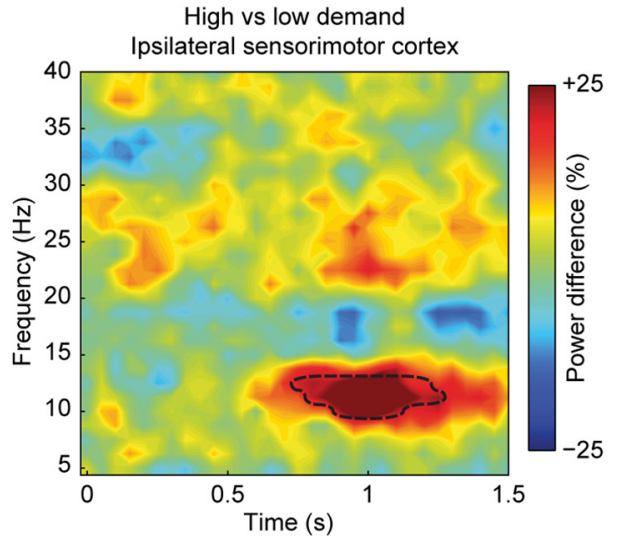

E

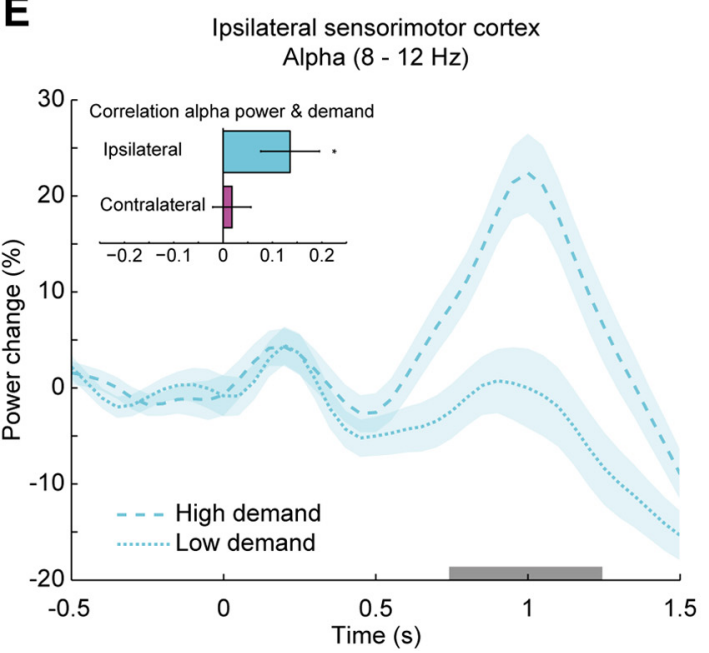

B

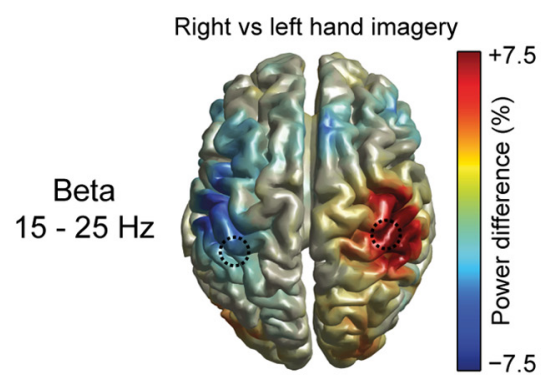

D

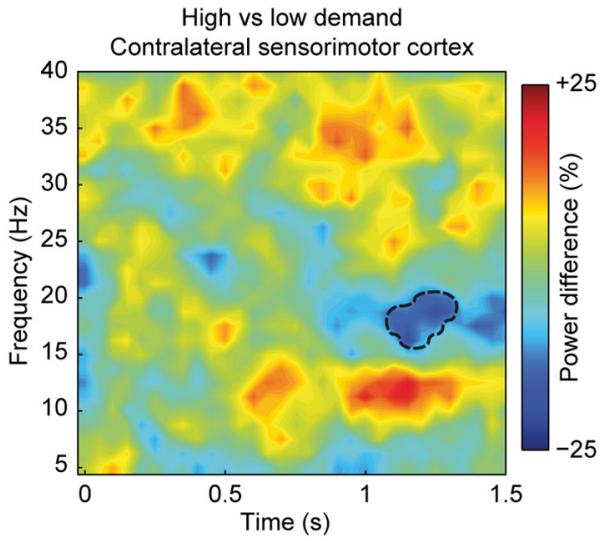

$\mathbf{F}$

Contralateral sensorimotor cortex Beta $(15-25 \mathrm{~Hz})$

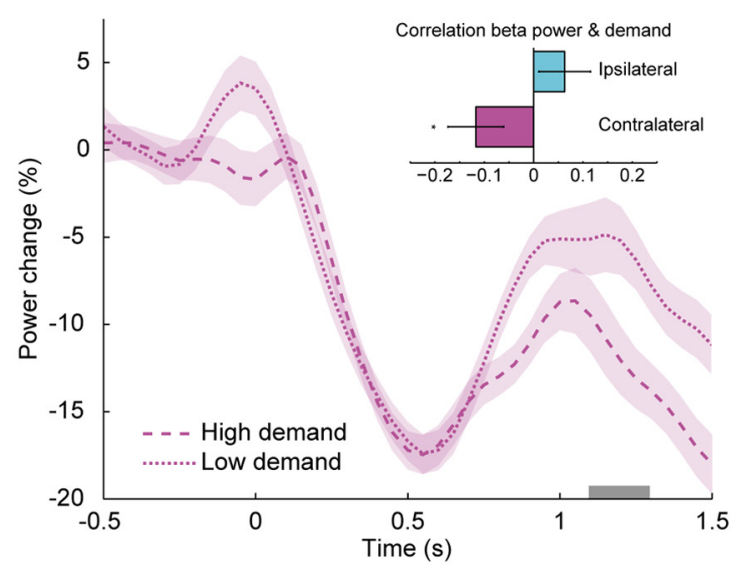

Figure 2. $A, B$, Source-reconstructed distribution of differential power changes between 600 and $1000 \mathrm{~ms}$ after stimulus presentation between motor imagery trials involving the right or left hand are shown for the alpha- and beta-band frequency, respectively. $\boldsymbol{C}, \boldsymbol{D}$, Dashed circles indicate the location of the voxels that were selected for subsequent analyses. The TFRs show the differential power changes measured during motor imagery with high and low movement selection demands. At the sensorimotor cortex ipsilateral to the hand that was used for imagery, there was a relative increase in alpha-band power in motor imagery trials with high selection demands compared with motor imagery trials with low selection demands (C). The sensorimotor cortex contralateral to the hand that was used for imagery showed a relative decrease in beta-band power (D). Black dashed lines mark the time-frequency boundaries of significant clusters. The line plots illustrate the temporal dynamics of the baseline-corrected power changes during motor imagery trials in the sensorimotor cortex ipsilateral $(\boldsymbol{E})$ or contralateral $(\boldsymbol{F})$ to the hand used for imagery. The gray bars along the $x$-axes indicate the time interval of the clusters shown in $\boldsymbol{C}$ and $\boldsymbol{D}$. Shaded areas indicate $\pm 0.5 \mathrm{~S}$ of the difference between conditions. The insets of $\boldsymbol{E}$ and $\boldsymbol{F}$ show the average Pearson correlation coefficients between task demand and alpha- and beta-band power, respectively (error bars represent $\pm 1 \mathrm{SE},{ }^{*} p<0.025$, one-sided $t$ test).

oscillatory power in the alpha-band is increased in the sensorimotor cortex ipsilateral to the hand used for imagery, whereas the oscillatory power in the beta-band is concurrently decreased in the contralateral hemisphere. This finding emerged within the motor imagery task, when trials with high selection demands were compared with trials with lower movement selection demands. The same pattern of power changes was observed when motor imagery trials were compared with control trials with identical stimuli and responses, but without imagery demands. In both analyses, the dissociation of alpha- and beta-band power was observed within the time frame of the simulated movements. This study provides novel empirical evidence for the notion that neural oscillations in the alpha- and beta-bands support the simulation of actions with different functional mechanisms, within a single paradigm. These findings are in line with previous theoretical suggestions (Hari and Salmelin, 1997; Leocani et al., 1997; 
A
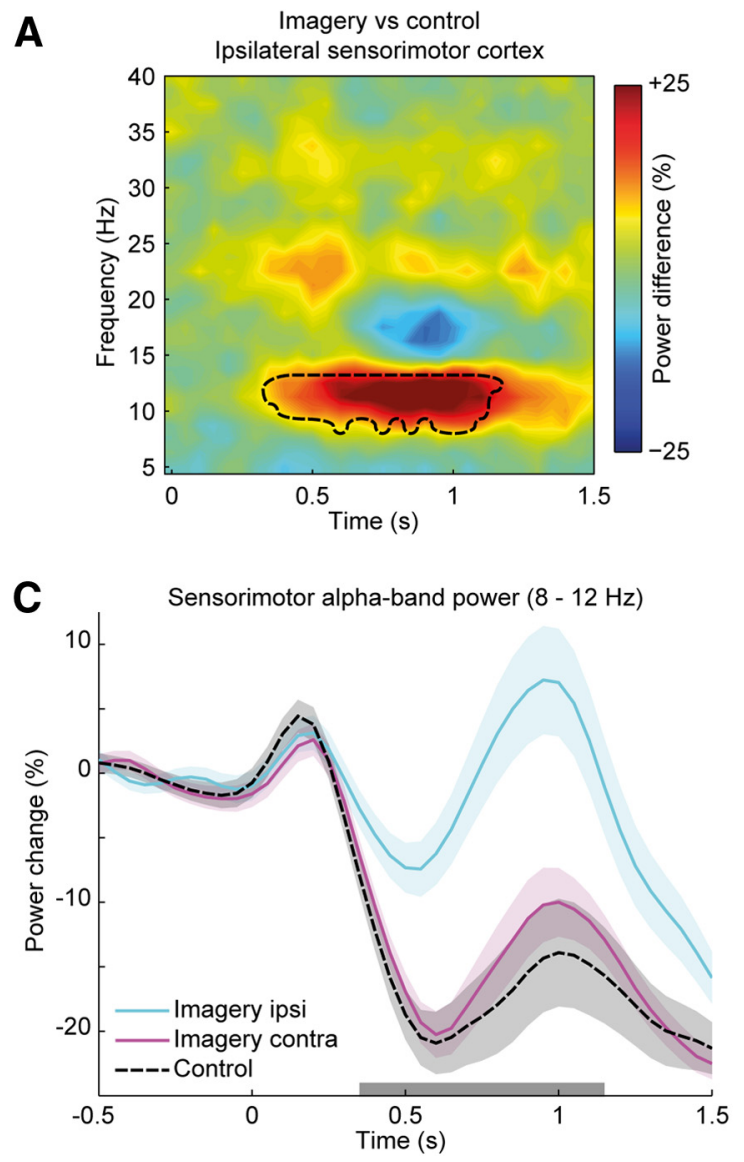

B
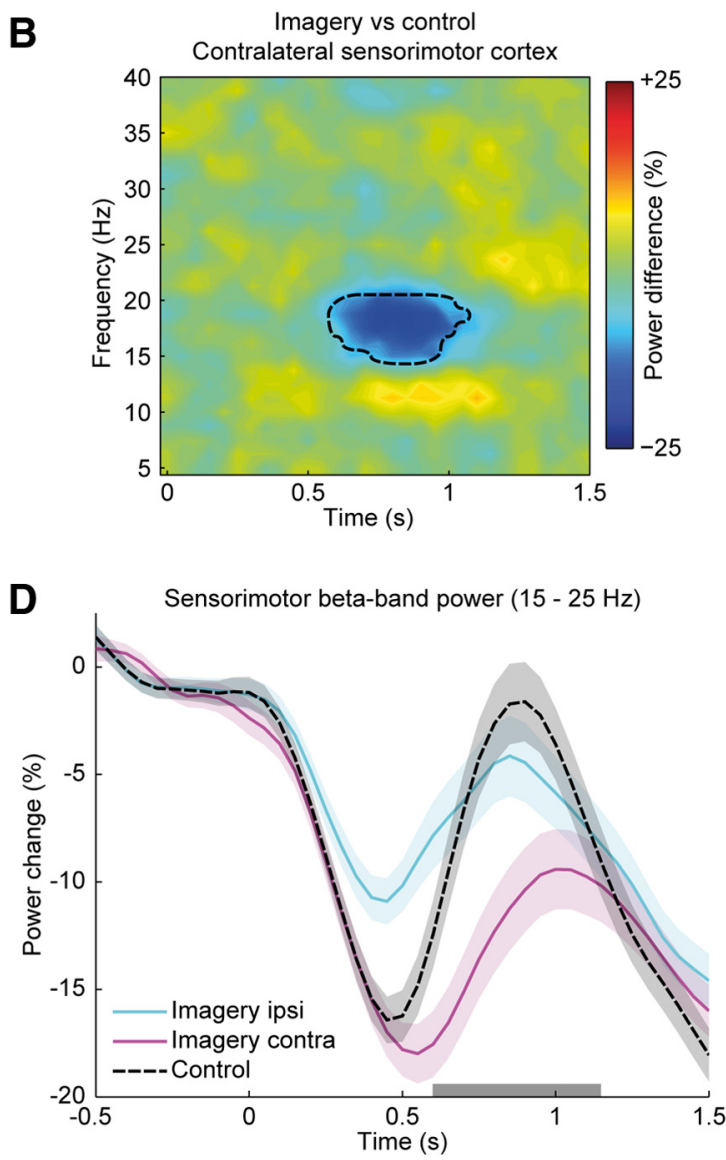

Figure 3. $\boldsymbol{A}, \boldsymbol{B}$, Source-reconstructed TFRs of differential power changes between imagery and control tasks are shown for the ipsilateral $(\boldsymbol{A})$ and contralateral sensorimotor cortices $(\boldsymbol{B})$. At the sensorimotor cortex ipsilateral to the hand that was used for imagery, there was a relative increase in alpha-band power in the motor imagery condition compared with the control condition $(\boldsymbol{A})$. The sensorimotor cortex contralateral to the hand that was used for imagery showed a relative decrease in beta-band power (B). Black dashed lines mark the time-frequency boundaries of significant clusters. $\boldsymbol{C}, \boldsymbol{D}$, The line plots illustrate baseline-corrected power changes of alpha- and beta-band power measured over sensorimotor cortices during imagery and control trials. The line plots distinguish between power changes evoked in the sensorimotor cortex ipsilateral ("imagery ipsi") and contralateral ("imagery contra") to the imagined hand. The black dashed lines indicate the spectral power during the control condition (averaged over left and right sensorimotor cortices). The gray bars along the $x$-axes indicate the time intervals of the clusters shown in $\boldsymbol{A}$ and $\boldsymbol{B}$. Shaded areas indicate $\pm 0.5 \mathrm{SE}$ of the differences between imagery and control trials.

Dipoppa and Gutkin, 2013), and call for a re-evaluation of the role of alpha- and beta-band rhythms in the sensorimotor system. We propose that neural oscillations in the alpha-band mediate the allocation of computational resources by disengaging task-irrelevant cortical regions. In contrast, the reduction of neural oscillations in the beta-band is directly related to the disinhibition of neuronal populations involved in the computations of movement parameters.

Previous studies investigating task-induced modulations of alpha- and beta-band power have not identified the dissociation shown in this study (Pfurtscheller and Lopes da Silva, 1999; de Lange et al., 2008; Bauer et al., 2012; ter Horst et al., 2013; Heinrichs-Graham et al., 2014). However, those studies did not investigate differential effects of task demand on spectral profiles nor included a neutral control condition. Without these manipulations, the temporal dynamics of spectral power evoked during motor imagery appears similar across alpha- and beta-bands (Fig. $3 C, D)$. This study indicates that the similarity of the lateralized pattern in the power distribution of alpha- and beta-band oscillations is superficial and arises from different computational mechanisms.

\section{The role of alpha-band oscillations in mental simulations}

We interpret the relative increase of alpha-band power in the ipsilateral hemisphere as the inhibition of neuronal ensembles interfering with current task requirements. This interpretation is in line with the notion that alpha-band oscillations implement "gating by inhibition" (Jensen and Mazaheri, 2010). Within this framework, stronger oscillations in the alpha-band are a mechanism for inhibiting neuronal processing in task-irrelevant cortical regions. This framework has been developed on the basis of lateralized power changes observed during selective spatial processing of sensory material, first in occipital and parietal regions (Worden et al., 2000; Thut et al., 2006; Tan et al., 2013), and then in the somatosensory system (Haegens et al., 2010, 2011, 2012; Jones et al., 2010; Anderson and Ding, 2011). This study generalizes this framework to sensorimotor processing, showing that during motor imagery the alpha-band power is selectively increased in the ipsilateral sensorimotor cortex.

The modulation of alpha-band power related to the simulation of the movements occurred predominantly in the ipsilateral sensorimotor cortex. This task-specific effect was embedded in a generic bilateral decrease of alpha-band power, which was present during both the imagery and the control condition. These findings show the lack of specificity intrinsic in the relative decrease in alpha-band power between baseline and task epochs. This finding has important consequences. It challenges the wideheld view that reduced alpha-band power in sensorimotor regions (or mu-rhythm desynchronization) can be used as a 
reliable marker of sensorimotor processing (Pfurtscheller and Lopes da Silva, 1999; Lepage et al., 2010; Stapel et al., 2010; Marshall and Meltzoff, 2011; Nyström et al., 2011; Reid et al., 2011; ter Horst et al., 2013). We suggest instead that a decrease in alphaband power is generally driven by processes unrelated to sensorimotor processes, e.g., power changes driven by the visual input or changes in arousal.

One might ask what is actually inhibited by the alpha-band power increase observed in this study. For instance, the increased alpha-band power could reflect the inhibition of a specific movement, namely an affordance of a grasping movement with the hand that was not used for imagery (intermanual conflict). However, participants did not need to acutely inhibit the irrelevant hand on each trial, given that the relevant hand was preselected over blocks of 10 subsequent trials. Moreover, if the increase in alpha-band power was driven by intermanual conflict, one would expect this effect to be especially prominent during the first trials of each block, where participants had just changed the hand they used for imagery. Additional analyses did not show such an effect for the first versus the last trials of each block (data not shown). Furthermore, alpha-band power increased even further when subjects selected between overhand and underhand grips that involved the same hand. These observations argue against the inhibition of a grasping movement with the nonselected hand. It seems more parsimonious to infer that the increase in alpha-band power implements a generic filtering mechanism for reducing the set of neuronal ensembles involved in the simulation of an action to reallocate computational resources.

\section{The role of beta-band oscillations in mental simulations}

The contralateral dominance of the beta-band power decrease and its modulation by task demand suggests that the power changes in this frequency band are directly related to the disinhibition of neuronal populations involved in the specification of motor parameters. These observations fit with the notion of betaband oscillations as an anti-kinetic rhythm that emerges throughout a network encompassing the cerebellum, basal ganglia, thalamus, spinal cord, and cortical sensorimotor regions (Brown, 2007; Engel and Fries, 2010; Jenkinson and Brown, 2011; van Ede and Maris, 2013). The functional relevance of the betaband rhythm in the disinhibition of neuronal populations becomes particularly clear in $\mathrm{PD}$, where pathological high betaband activity severely compromises movement initiation and execution (Brown, 2007; Jenkinson and Brown, 2011). Stimulation of the subthalamic nucleus at the beta-band frequency further enhanced these symptoms in PD patients (Chen et al., 2007), suggesting a causal role between beta-band activity and the disinhibition of these neuronal populations. Similar results have been found when rhythmic activity was induced in the motor cortex of healthy participants using transcranial current stimulation. Stimulation in the beta-band frequency range was particularly effective in slowing movements and increasing the threshold of inducing a motor response (Pogosyan et al., 2009; Feurra et al., 2011; Wach et al., 2013). However, suppression of beta-band activity per se is unlikely to be directly related to the computations of movement parameters. For instance, in previous studies on the preparation of movements of different hand configuration, force and/or direction could not be discriminated based on their beta-band activity (Rickert et al., 2005; Waldert et al., 2008; Zaepffel et al., 2013). We therefore propose that the reduction of beta-band power weakens inhibition over the sensorimotor cortex, allowing neuronal populations to converge into a state space suitable to initiate an action (Churchland et al., 2010; Kaufman et al., 2014). This interpretation is in line with the observation that stimulus orientations affording grasping movements with both underhand and overhand grasps were associated with a stronger and longer decrease in beta-band power. In those trials, a larger neuronal search space needs to spanned (Cisek and Kalaska, 2010), implying a stronger and longer disinhibition through beta-band suppression. A similar modulation of beta-band power was observed by Grent-'t-Jong et al. (2013) where betaband was stronger when competition between multiple possible actions needed to be resolved. These observations suggest that enhanced beta suppression is a relevant but indirect marker of selection between action plans, and different from the inhibitory mechanisms supported by oscillations in the alpha-band.

\section{Interpretational issues}

It remains unclear why both ipsilateral alpha-band power enhancement and contralateral beta-band power suppression emerged relatively late during the imagery epoch $(>500 \mathrm{~ms}$ after stimulus presentation). One would expect this differential modulation to be present from the onset of the simulation of the movement, e.g., when the lateralization of alpha and beta lateralization becomes apparent ( $\sim 250 \mathrm{~ms}$ after stimulus presentation). Early aspects of these modulations in oscillatory power might have been statistically obscured by intersubject variability, and more sensitive electrophysiological methods (e.g., electrocorticography) might help to better characterize the early dynamics of alpha- and beta-band power changes during motor imagery. Another issue is that the modulation of oscillatory power as a function of task demands emerges later than the differential effect between imagery and control trials. These numerical differences indicate that high- and low-demand trials share an initial phase where they both show a similar difference in spectral power relative to the control task, which is further sustained and enhanced in the high-demand trials. The prolonged duration of the electrophysiological effect matches the prolongation of the imagery process with increasing selection demands, in line with the longer reaction times evoked during high-demand trials.

Similar lateralized power changes in the alpha- and/or betabands have been shown in studies of somatosensory attention toward anticipated sensory inputs (Jones et al., 2010; Anderson and Ding, 2011; van Ede et al., 2011), and prediction of the somatosensory consequences of a movement is an intrinsic element of a motor simulation (Wolpert, 1997; Davidson and Wolpert, 2005; Shadmehr and Krakauer, 2008). However, a number of empirical and theoretical considerations argue against the possibility that the power changes reported in this study are directly related to anticipation of impending tactile information or somatosensory attention. First, during the imagery task, participants did not anticipate actual somatosensory feedback. Accordingly, there were no power changes in the prestimulus baseline period as a function of which hand was used for imagery. This suggests that the observed power changes are not driven by somatosensory attention to the hand used for imagery. Second, power changes related to directing somatosensory attention to one hand or the other are not expected to be modulated by task demands, because attention would be matched across high- and low-demand trials. As both the alpha-band power in the ipsilateral sensorimotor cortex and the beta-band power in the contralateral sensorimotor cortex were modulated by task demands, these changes cannot be explained by directing somatosensory attention. 


\section{Conclusions}

Our current observations extend to the sensorimotor domain the notion that alpha-band oscillations support the disengagement of task-irrelevant cortical regions (Jensen and Mazaheri, 2010), whereas a reduction in beta-band power allows specific sensorimotor neuronal ensembles to coordinate their computations of movement parameters (Brown, 2007; Jenkinson and Brown, 2011). These findings contribute to a more refined understanding of the role of oscillatory rhythms in the sensorimotor system.

\section{References}

Anderson KL, Ding M (2011) Attentional modulation of the somatosensory mu rhythm. Neuroscience 180:165-180. CrossRef Medline

Bauer M, Kennett S, Driver J (2012) Attentional selection of location and modality in vision and touch modulates low-frequency activity in associated sensory cortices. J Neurophysiol 107:2342-2351. CrossRef Medline

Brown P (2000) Cortical drives to human muscle: the piper and related rhythms. Prog Neurobiol 60:97-108. CrossRef Medline

Brown P (2007) Abnormal oscillatory synchronisation in the motor system leads to impaired movement. Curr Opin Neurobiol 17:656-664. CrossRef Medline

Carlqvist H, Nikulin VV, Strömberg JO, Brismar T (2005) Amplitude and phase relationship between alpha and beta oscillations in the human electroencephalogram. Med Biol Eng Comput 43:599-607. CrossRef Medline

Chen CC, Litvak V, Gilbertson T, Kühn A, Lu CS, Lee ST, Tsai CH, Tisch S, Limousin P, Hariz M, Brown P (2007) Excessive synchronization of basal ganglia neurons at $20 \mathrm{~Hz}$ slows movement in Parkinson's disease. Exp Neurol 205:214-221. CrossRef Medline

Cheyne DO (2013) MEG studies of sensorimotor rhythms: a review. Exp Neurol 245:27-39. CrossRef Medline

Churchland MM, Cunningham JP, Kaufman MT, Ryu SI, Shenoy KV (2010) Cortical preparatory activity: representation of movement or first cog in a dynamical machine? Neuron 68:387-400. CrossRef Medline

Cisek P, Kalaska JF (2010) Neural mechanisms for interacting with a world full of action choices. Annu Rev Neurosci 33:269-298. CrossRef Medline

Crone NE, Miglioretti DL, Gordon B, Lesser RP (1998) Functional mapping of human sensorimotor cortex with electrocorticographic spectral analysis. II. Event-related synchronization in the gamma band. Brain 121: 2301-2315. CrossRef Medline

Davidson PR, Wolpert DM (2005) Widespread access to predictive models in the motor system: a short review. J Neural Eng 2:S313-9. CrossRef Medline

de Lange FP, Jensen O, Bauer M, Toni I (2008) Interactions between posterior gamma and frontal alpha/beta oscillations during imagined actions. Front Hum Neurosci 2:7. CrossRef Medline

Dipoppa M, Gutkin BS (2013) Flexible frequency control of cortical oscillations enables computations required for working memory. Proc Natl Acad Sci U S A 110:12828-12833. CrossRef Medline

Engel AK, Fries P (2010) Beta-band oscillations-signalling the status quo? Curr Opin Neurobiol 20:156-165. CrossRef Medline

Feurra M, Bianco G, Santarnecchi E, Del Testa M, Rossi A, Rossi S (2011) Frequency-dependent tuning of the human motor system induced by transcranial oscillatory potentials. J Neurosci 31:12165-12170. CrossRef Medline

Grent-'t-Jong T, Oostenveld R, Jensen O, Medendorp WP, Praamstra P (2013) Oscillatory dynamics of response competition in human sensorimotor cortex. Neuroimage 83:27-34. CrossRef Medline

Gross J, Kujala J, Hamalainen M, Timmermann L, Schnitzler A, Salmelin R (2001) Dynamic imaging of coherent sources: studying neural interactions in the human brain. Proc Natl Acad Sci U S A 98:694-699. CrossRef Medline

Haegens S, Osipova D, Oostenveld R, Jensen O (2010) Somatosensory working memory performance in humans depends on both engagement and disengagement of regions in a distributed network. Hum Brain Mapp 31:26-35. CrossRef Medline

Haegens S, Händel BF, Jensen O (2011) Top-down controlled alpha band activity in somatosensory areas determines behavioral performance in a discrimination task. J Neurosci 31:5197-5204. CrossRef Medline

Haegens S, Luther L, Jensen O (2012) Somatosensory anticipatory alpha activity increases to suppress distracting input. J Cogn Neurosci 24:677685. CrossRef Medline

Hari R, Salmelin R (1997) Human cortical oscillations: a neuromagnetic view through the skull. Trends Neurosci 20:44-49. CrossRef Medline

Heinrichs-Graham E, Wilson TW, Santamaria PM, Heithoff SK, TorresRussotto D, Hutter-Saunders JA, Estes KA, Meza JL, Mosley RL, Gendelman HE (2014) Neuromagnetic evidence of abnormal movement-related beta desynchronization in Parkinson's disease. Cereb Cortex 24:2669-2678. CrossRef Medline

Jeannerod M (1994) The representing brain: neural correlates of motor intention and imagery. Behav Brain Sci 17:187-202. CrossRef

Jeannerod M, Decety J (1995) Mental motor imagery: a window into the representational stages of action. Curr Opin Neurobiol 5:727-732. CrossRef Medline

Jenkinson N, Brown P (2011) New insights into the relationship between dopamine, beta oscillations and motor function. Trends Neurosci 34: 611-618. CrossRef Medline

Jensen O, Mazaheri A (2010) Shaping functional architecture by oscillatory alpha activity: gating by inhibition. Front Hum Neurosci 4:186. CrossRef Medline

Jones SR, Kerr CE, Wan Q, Pritchett DL, Hämäläinen M, Moore CI (2010) Cued spatial attention drives functionally relevant modulation of the mu rhythm in primary somatosensory cortex. J Neurosci 30:13760-13765. CrossRef Medline

Jurkiewicz MT, Gaetz WC, Bostan AC, Cheyne D (2006) Post-movement beta rebound is generated in motor cortex: evidence from neuromagnetic recordings. Neuroimage 32:1281-1289. CrossRef Medline

Kaufman MT, Churchland MM, Ryu SI, Shenoy KV (2014) Cortical activity in the null space: permitting preparation without movement. Nat Neurosci 17:440-448. CrossRef Medline

Kilner JM, Baker SN, Salenius S, Hari R, Lemon RN (2000) Human cortical muscle coherence is directly related to specific motor parameters. J Neurosci 20:8838-8845. Medline

Leocani L, Toro C, Manganotti P, Zhuang P, Hallett M (1997) Event-related coherence and event-related desynchronization/synchronization in the $10 \mathrm{~Hz}$ and $20 \mathrm{~Hz}$ EEG during self-paced movements. Electroencephalogr Clin Neurophysiol 104:199-206. CrossRef Medline

Lepage JF, Tremblay S, Nguyen DK, Champoux F, Lassonde M, Théoret H (2010) Action related sounds induce early and late modulations of motor cortex activity. Neuroreport 21:250-253. CrossRef Medline

Liljeström M, Kujala J, Jensen O, Salmelin R (2005) Neuromagnetic localization of rhythmic activity in the human brain: a comparison of three methods. Neuroimage 25:734-745. CrossRef Medline

Mackay WA (1997) Synchronized neuronal oscillations and their role in motor processes. Trends Cogn Sci 1:176-183. CrossRef Medline

Maris E (2012) Statistical testing in electrophysiological studies. Psychophysiology 49:549-565. CrossRef Medline

Maris E, Oostenveld R (2007) Nonparametric statistical testing of EEG- and MEG-data. J Neurosci Methods 164:177-190. CrossRef Medline

Marshall PJ, Meltzoff AN (2011) Neural mirroring systems: exploring the EEG mu rhythm in human infancy. Dev Cogn Neurosci 1:110-123. CrossRef Medline

McFarland DJ, Miner LA, Vaughan TM, Wolpaw JR (2000) Mu and beta rhythm topographies during motor imagery and actual movements. Brain Topogr 12:177-186. CrossRef Medline

Mima T, Hallett M (1999) Corticomuscular coherence: a review. J Clin Neurophysiol 16:501-511. CrossRef Medline

Nierula B, Hohlefeld FU, Curio G, Nikulin VV (2013) No somatotopy of sensorimotor alpha-oscillation responses to differential finger stimulation. Neuroimage 76:294-303. CrossRef Medline

Nolte G (2003) The magnetic lead field theorem in the quasi-static approximation and its use for magnetoencephalography forward calculation in realistic volume conductors. Phys Med Biol 48:3637-3652. CrossRef Medline

Nyström P, Ljunghammar T, Rosander K, von Hofsten C (2011) Using mu rhythm desynchronization to measure mirror neuron activity in infants. Dev Sci 14:327-335. CrossRef Medline

Oostenveld R, Fries P, Maris E, Schoffelen JM (2011) FieldTrip: open source software for advanced analysis of MEG, EEG, and invasive electrophysiological data. Comput Intell Neurosci 2011:156869. CrossRef Medline 
Pfurtscheller G, Lopes da Silva FH (1999) Event-related EEG/MEG synchronization and desynchronization: basic principles. Clin Neurophysiol 110:1842-1857. CrossRef Medline

Pogosyan A, Gaynor LD, Eusebio A, Brown P (2009) Boosting cortical activity at Beta-band frequencies slows movement in humans. Curr Biol 19:1637-1641. CrossRef Medline

Reid VM, Striano T, Iacoboni M (2011) Neural correlates of dyadic interaction during infancy. Dev Cogn Neurosci 1:124-130. CrossRef Medline

Rickert J, Oliveira SC, Vaadia E, Aertsen A, Rotter S, Mehring C (2005) Encoding of movement direction in different frequency ranges of motor cortical local field potentials. J Neurosci 25:8815-8824. CrossRef Medline

Rosenbaum DA, Jorgensen MJ (1992) Planning macroscopic aspects of manual control. Hum Mov Sci 11:61-69. CrossRef

Salmelin R, Hämäläinen M, Kajola M, Hari R (1995) Functional segregation of movement-related rhythmic activity in the human brain. Neuroimage 2:237-243. CrossRef Medline

Sanes JN, Donoghue JP (1993) Oscillations in local field potentials of the primate motor cortex during voluntary movement. Proc Natl Acad Sci U S A 90:4470-4474. CrossRef Medline

Shadmehr R, Krakauer JW (2008) A computational neuroanatomy for motor control. Exp Brain Res 185:359-381. CrossRef Medline

Stapel JC, Hunnius S, van Elk M, Bekkering H (2010) Motor activation during observation of unusual versus ordinary actions in infancy. Soc Neurosci 5:451-460. CrossRef Medline

Stolk A, Todorovic A, Schoffelen JM, Oostenveld R (2013) Online and offline tools for head movement compensation in MEG. Neuroimage 68: 39-48. CrossRef Medline

Tan HRM, Leuthold H, Gross J (2013) Gearing up for action: attentive tracking dynamically tunes sensory and motor oscillations in the alpha and beta band. Neuroimage 82:634-644. CrossRef Medline

ter Horst AC, van Lier R, Steenbergen B (2013) Mental rotation strategies reflected in event-related (de)synchronization of alpha and mu power. Psychophysiology 50:858-863. CrossRef Medline

Thut G, Nietzel A, Brandt SA, Pascual-Leone A (2006) Alpha-band electroencephalographic activity over occipital cortex indexes visuospatial atten- tion bias and predicts visual target detection. J Neurosci 26:9494-9502. CrossRef Medline

van Ede F, Maris E (2013) Somatosensory demands modulate muscular beta oscillations, independent of motor demands. J Neurosci 33:10849_ 10857. CrossRef Medline

van Ede F, de Lange F, Jensen O, Maris E (2011) Orienting attention to an upcoming tactile event involves a spatially and temporally specific modulation of sensorimotor alpha- and beta-band oscillations. J Neurosci 31:2016-2024. CrossRef Medline

van Veen BD, van Drongelen W, Yuchtman M, Suzuki A (1997) Localization of brain electrical activity via linearly constrained minimum variance spatial filtering. IEEE Trans Biomed Eng 44:867-880. CrossRef Medline

van Wijk BCM, Beek PJ, Daffertshofer A (2012) Neural synchrony within the motor system: what have we learned so far? Front Hum Neurosci 6:252. CrossRef Medline

Wach C, Krause V, Moliadze V, Paulus W, Schnitzler A, Pollok B (2013) Effects of $10 \mathrm{~Hz}$ and $20 \mathrm{~Hz}$ transcranial alternating current stimulation (tACS) on motor functions and motor cortical excitability. Behav Brain Res 241:1-6. CrossRef Medline

Waldert S, Preissl H, Demandt E, Braun C, Birbaumer N, Aertsen A, Mehring C (2008) Hand movement direction decoded from MEG and EEG. J Neurosci 28:1000-1008. CrossRef Medline

Wolpert DM (1997) Computational approaches to motor control. Trends Cogn Sci 1:209-216. CrossRef Medline

Wolpert DM, Ghahramani Z (2000) Computational principles of movement neuroscience. Nat Neurosci [3 Suppl]:1212-1217. CrossRef Medline

Wood DK, Goodale MA (2011) Selection of wrist posture in conditions of motor ambiguity. Exp brain Res 208:607-620. CrossRef Medline

Worden MS, Foxe JJ, Wang N, Simpson GV (2000) Anticipatory biasing of visuospatial attention indexed by retinotopically specific alpha-band electroencephalography increases over occipital cortex. J Neurosci 20:RC63. Medline

Zaepffel M, Trachel R, Kilavik BE, Brochier T (2013) Modulations of EEG beta power during planning and execution of grasping movements. PLoS One 8:e60060. CrossRef Medline 